\title{
Universal newborn hearing screening: methods and results, obstacles, and benefits
}

\author{
Katarzyna E. Wroblewska-Seniuk' ${ }^{1}$, Piotr Dabrowski ${ }^{2}$, Witold Szyfter ${ }^{2}$ and Jan Mazela ${ }^{1}$
}

\begin{abstract}
The incidence of sensorineural hearing loss ranges from 1 to 3 per 1,000 live births in term healthy neonates, and 2-4 per 100 in high-risk infants, a 10-fold increase. Early identification and intervention with hearing augmentation within 6 mo yields optimal effect. If undetected and without treatment, significant hearing impairment may negatively impact speech development and lead to disorders in psychological and mental behaviors. Hearing screening programs in newborns enable detection of hearing impairment in the first days after birth. Programs to identify hearing deficit have significantly improved over the two decades, and their implementation continues to grow throughout the world. Initially based on risk factors, these programs identified only $50-75 \%$ of infants with hearing loss. Current recommendations are to conduct universal hearing screening in all infants. Techniques used primarily include automated auditory brainstem responses and otoacoustic emissions that provide noninvasive recordings of physiologic auditory activity and are easily performed in neonates and infants. The aim of this review is to present the objectives, benefits, and results of newborn hearing screening programs including the pros and cons of universal vs. selective screening. A brief history and the anticipated future development of these programs will also be discussed.
\end{abstract}

C hildhood hearing impairment is the result of the overlapping factors of genetic predisposition, the intrauterine environment, perinatal, and postnatal factors. Worldwide reporting of hearing loss finds that the prevalence of moderate and severe bilateral hearing deficit $(>40 \mathrm{~dB})$ is $1-3$ per 1,000 live births in well baby nursery population $(1,2)$ and $2-4$ in 100 infants in an intensive care population (3-6). The numbers given above signify that hearing impairment is one of the most common potentially disabling conditions present in infancy and one of the most frequent congenital anomalies (7-9).

The definition of hearing loss and hearing deficit may vary in different classification systems but usually categories of hearing loss are mild (21-40 dB HL), moderate (41-70 dB HL), severe (71-95 dB HL), and profound ( $>95 \mathrm{~dB} \mathrm{HL})$. Deafness is the term reserved for profound hearing loss (10). Thresholds are expressed in $\mathrm{dB}$ on the hearing level scale $(\mathrm{dB} H \mathrm{HL})$.
Exposure to spoken language is vital during early childhood. Therefore, children with undiagnosed hearing loss, including mild and unilateral deficit can experience considerable delays in speech development and present with disorders in psychological and mental behavior which can influence their social and academic skills (11-13).

Universal newborn hearing screening (UNHS) is a strategy that enables to identify congenital deafness and hearing loss. Over the past two decades, screening neonates for hearing deficit has become the standard of care in many countries all over the world. The major objective of UNHS is to identify children with all kinds and degrees of hearing impairment, both bilateral and unilateral and to lower the age at the time of diagnosis for early hearing amplification, to maximize their linguistic competence and literacy development (14). Yoshinga Itano reported that at $2 \mathrm{y}$ of age infants with hearing loss, identified before the age of $6 \mathrm{mo}$, had better mean scores in expressive and receptive language than those identified later $(4,15,16)$.

\section{ETIOLOGY OF NEONATAL HEARING IMPAIRMENT}

There are many known causes of neonatal hearing loss. It is generally accepted that genetic and environmental factors are each responsible for half of the cases of congenital hearing impairment (17). Among genetic defects $30 \%$ of causes are considered syndromic, whereas $70 \%$ is thought to be nonsyndromic and about half of them are presumed to be due to a mutation in the connexin encoding gene (18).

The nongenetic causes of hearing impairment can be divided into prenatal, perinatal, postnatal, occasional, and unknown.

Prenatal origins of hearing deficit include many congenital infections and nongenetic congenital malformations. Cytomegalovirus infection with a prevalence of around 0.2$2.5 \%$ of all neonates worldwide is said to be the main cause of nongenetic hearing loss in children. Both symptomatic and nonsymptomatic congenital cytomegalovirus infections can lead to hearing impairment, commonly occurring after the newborn period. The hearing loss of this etiology is mostly sensorineural and profound, and can also be progressive (19). Similarly, other TORCH infections, such as toxoplasmosis, syphilis, rubella, and herpes are reportedly associated with 


\section{Review | Wroblewska-Seniuk et al.}

neonatal hearing impairment, although treatment for congenital toxoplasmosis and syphilis, as well as rubella vaccine have significantly reduced the risk of hearing loss (20). It has been recently proven that also Zika virus, which is known to cause microcephaly in fetus and neonates, might be an etiological factor of hearing loss in newborns and young children. Leal et al. have shown that the prevalence of sensorineural hearing loss in children with Zika virus infection was $5.8 \%$, which is similar to that seen in association with other congenital viral infections (21). The American Academy of Audiology suggests that hearing loss due to the Zika Virus Disease can occur at birth or be acquired later and therefore it is crucially important to identify hearing loss through infant hearing screening or preschool and school-aged screening programs for all children who may be at risk as a consequence of Zika virus disease (22). Follow-up evaluation of hearing is also recommended for newborns with congenital cytomegalovirus infection, toxoplasmosis, rubella or syphilis.

Perinatal causes of hearing loss include prematurity, low Apgar scores and hyperbilirubinemia with kernicterus. Additionally, prematurity might be associated with such risk factors of hearing loss as: ototoxic drugs-aminoglycosides and loop diuretics, noise exposure during treatment in neonatal intensive care unit (NICU) and hypoxia.

Aminoglycosides in association with $\beta$-lactams are often used as the first line antibiotic treatment in newborns and are widely used in NICUs. Unfortunately, they are known to damage both cochlear and vestibular organs. Gentamycin and tobramycin are the most vestibulotoxic agents, whereas neomycin, kanamycin, and amikacin are more toxic toward cochlea. These drugs produce irreversible hearing loss by causing hair cell death. The damage to hair cells from aminoglycosides affects initially high-frequency hearing and progresses to lower frequencies $(23,24)$. Ototoxicity of aminoglycoside correlates with duration of treatment, high peak and trough concentrations and concurrent administration of other drugs, such as loop diuretics and vancomycin. Loop diuretics alone produce reversible hearing loss by inhibiting ion transport within stria vascularis in cochlea. They increase also the rate and severity of aminoglycoside-induced permanent hearing loss causing alterations in the blood-labyrinthine barrier, which facilitates aminoglycoside entry into the endolymphatic fluid compartment (23).

Environmental factors also play an important role in potential hearing problems. Exposure to the constant background noise generated by contemporary life-support equipment in the NICU can lead to hearing loss. The first effect of noise is outer hair cell damage which is seen in the results of otoacoustic emission (OAE) and automated auditory brain stem response (AABR). It is recommended by the American Academy of Pediatrics that the average noise levels in neonatal units should be below $45 \mathrm{~dB}$ in infants' areas and that transient sounds should not exceed $65 \mathrm{~dB}(23,25-27)$. Particularly dangerous for newborns are hazardous sound levels during transport. For example, in an ambulance or helicopter the noise level can reach 90-110 dB. The main defense against noise for neonates are incubators, which usually reduce the sounds by 5-18 dB $(26,27)$.

Hyperbilirubinemia which is widely known to be a risk factor for hearing loss can cause selective damage to the brainstem auditory nuclei and may damage the auditory nerve and ganglion cells, while there is no evidence of damage at the level of the cochlea (28). Auditory neural damage from bilirubin toxicity ranges from neural timing deficits, including dyssynchrony, to neural response reduction and elimination of auditory neural responses. This condition is comprehensively described as auditory neuropathy spectrum disorder (ANSD) $(28,29)$.

Hypoxia has strong association with hearing loss, as adequate oxygenation and perfusion are essential for normal cochlear function (30). It is known that severe hypoxia may cause irreversible cellular damage to the outer hair cells and stria vascularis in the cochlea, however there is no clear threshold level of hypoxia at which hearing is at risk (30). Low Apgar scores $0-4$ at $1 \mathrm{~min}$ or $0-6$ at $5 \mathrm{~min}$ after birth have been used as indicators of birth asphyxia that has been associated with sensorineural hearing loss (20).

Additionally, Robertson et al. showed that mechanical ventilation and prolonged oxygen supplementation were associated with high prevalence of permanent hearing loss in extremely premature infants (31). Hille et al. similarly presented that assisted ventilation $\geq 5 \mathrm{~d}$ is an independent risk factor for hearing loss (6).

It is known from many studies that some NICU graduates may begin to develop hearing loss at the age of $2-4$ y (32). The pathophysiology of this delayed process is unclear, however, it may be caused by demyelination or degeneration at points along the auditory pathway (32).

\section{HISTORY OF HEARING SCREENING}

The initiation of screening for hearing defects can largely be attributed to the audiologist Marion Downs, as long ago as 1964 (33). During a remarkable career for more than five decades, Downs was proving the importance of early identification of hearing loss and the need to begin early interventions for babies with hearing deficits to help them with speech, language, and educational development. Pointing out the importance of the early infant hearing screening was not an easy task, because this procedure was not acceptable in the beginning by most pediatricians and otolaryngologists $(34,35)$.

At the beginning screening was performed in some countries by using the behavioral distraction test when the infant was 6-9 mo old. However, it has been shown in many studies that only children identified with hearing impairment early and provided with hearing amplification prior to 6 mo of age had a better chance of developing skills equivalent to their peers $(4,16,34)$. The discovery of OAEs enabled development of an easy and effective method for hearing screening in infants in the first days of life.

Initially newborn hearing screening was targeted toward newborns at risk for hearing deficit. In 1994 the Joint Committee on Infant Hearing (JCIH) listed 10 factors that identify infants at greatest risk for hearing impairment 
(Table 1) (36). Some studies reported additional risk factors such as admission to intensive care unit, premature birth, respiratory distress syndrome and intracranial hemorrhage $(3,6,37,38)$.

Many hearing screening programs in different countries comprised initially only children with specific risk factors. However, the selective screening of children resulted in $\sim 50 \%$ of all children with hearing loss being missed $(2,39,40)$. Therefore, it is now recommended to conduct universal hearing screening in all infants $(8,14)$.

\section{METHODS AND PROTOCOLS OF HEARING SCREENING}

The techniques most often employed and successfully used in the universal neonatal hearing screens are: (i) AABR and (ii) OAEs. Both OAE and AABR technologies provide noninvasive recordings of physiologic activity underlying normal auditory function and both are easily performed in neonates and infants (41). They are validated by professional organizations as reliable and objective screening methods $(1,14)$.

OAEs recording usually takes $<1 \mathrm{~min}$ and can be achieved without audiological expertise $(2,41)$. The principle of the test is that the sound vibrations emitted from the normal cochlear amplifier flow to the ear canal where the acoustic energy is recorded. During screening a small probe is placed in the ear canal, which delivers sound stimuli into the auditory system. In a healthy ear, the sound stimuli are transmitted through the middle ear to the inner ear where the outer hair cells of the cochlea produce an active response or emissions (42). These emissions are picked up by a microphone in the probe, analyzed by the screening unit and an automated "pass" or "refer" result is displayed on the unit screen.

Currently, two types of OAE measurements are used for newborn hearing screening: transient evoked OAEs and distortion product OAEs. Transient evoked OAEs represent the sum of pulse responses of outer hair cells along the cochlea, whereas distortion product OAEs arise directly from the frequency-selective compressive nonlinearity of outer hair cells amplifiers. Transient evoked OAEs more qualitatively assess cochlear function and are more suited for topologic diagnostics, while distortion product OAEs provide quantitative information about the hearing loss. In principle, both distortion product OAEs and transient evoked OAEs allow acquisition of frequency-specific information about a hearing loss problem (43). OAE can be used to assess cochlear function in the frequency range $500-6,000 \mathrm{~Hz}$.

OAE screening is highly sensitive (between 85 and 100\%) and reasonably specific (between 91 and 95\%) $(42,44,45)$. Basing on the analysis of multistep screening protocol of 4,519 children $0-3$ y of age, Eiserman et al. estimated the positive predictive value of OAE to be $67.3 \%$ and the estimated negative yield was calculated at $98.9 \%$ (46). However, the problem with OAE testing is the high referral rate to audiologic centers $(47,48)$. The JCIH guidelines require that a good UNHS program should have referral rates of no more than $4 \%$ (14). Given the prevalence of severe hearing deficit is $\sim 1-3$ per 1,000 live births in a well-baby nursery population, a referral rate of $4 \%$ means that 10 newborns are referred to second stage of the program for every case diagnosed with hearing impairment. It means that there are many false positive results which increase costs and workload of the program.

According to many studies, the main reason for falsepositive outcomes with OAE testing are transient conditions in the external auditory canal (e.g., collapse of the ear canal and the presence of debris) and middle ear (e.g., presence of amniotic fluid and mucus), as well as high ambient noise level (49). These problems usually resolve within the first few hours or days of life, and if the screening protocol involves more than one OAE tests more newborn pass it and the referral rate is lower.

Another reason for high referral rate are lower frequencies $(1-4 \mathrm{kHz})$ used in some OAE tests (49). Sounds of different frequencies are transmitted differently through the middle ear. The presence of amniotic fluid and mesenchyme in the middle ear in the first days of life reduces the volume of middle-ear air space and increases its stiffness, which affects the transmission of lower-frequency sounds. Screening involving higher frequencies $(2-4$ or $2-5 \mathrm{kHz})$ has lower referral rates as it is not that much affected by the presence of liquid and debris $(49,50)$.

However, as OAE are generated within the cochlea, OAE technology cannot be used to detect neural (eighth nerve and auditory brainstem pathway) dysfunction (40), that may result from exposure to ototoxic drugs or hyperbilirubinemia. To

Table 1. Risk factors for hearing impairment (JClH) (33)

1. Family history of hearing loss

2. Congenital infections

3. Craniofacial anomalies

4. Low birth weight $(<1,500 \mathrm{~g})$

5. Hyperbilirubinemia at serum level requiring exchange transfusion

6. Ototoxic medications (aminoglycosides in multiple courses and/or in combination with loop diuretics)

7. Bacterial meningitis

8. Low Apgar scores (0-4 at 1 min or $0-6$ at $5 \mathrm{~min}$ )

9. Mechanical ventilation for at least $5 \mathrm{~d}$

10. Syndromes associated with congenital hearing loss_-Usher syndrome, Pendred syndrome, Jervell and Lange-Nielsen syndrome, biotinidase deficiency, Refsum syndrome, Alport syndrome, Waardenburg syndrome, CHARGE syndrome, neurofibromatosis, and osteopetrosis $(10,19)$ 


\section{Review $\quad$ Wroblewska-Seniuk et al.}

diagnose and distinguish such pathology auditory brainstem responses are used.

Auditory brainstem response is an auditory evoked potential that originates from the auditory nerve. It can detect impairment on the level of cochlea, auditory nerve, and auditory pathway in the brainstem. AABR measurements are obtained by placing disposable surface electrodes on the forehead and recording brain wave activity in response to sound. The normal AABR takes the form of five successive neural waves labeled I-V, where wave I is the compound action potential of the peripheral portion of the cochlear nerve and wave $\mathrm{V}$ is generated in the mesencephalon. An infant's waveform is compared with the template of standard AABR infant data and "pass" or "fail" result is determined.

AABR plays a great role in testing site of the lesion-it enables the distinction between conductive and cochlear hearing loss. This method is highly sensitive to detect pathological mechanisms inducing hearing problems through disruption of afferent impulses, which is known as auditory neuropathy spectrum disorders. Presence of OAEs and concomitant major AABR abnormalities usually signify auditory neuropathy. On the contrary absence of OAE and preservation of normal AABR recordings enables to correct the diagnosis of hearing loss and usually signifies problems in middle ear (10). Table 2 shows the most important differences between OAE and AABR methods.
It is now recommended that infants admitted to the NICU for more than $5 \mathrm{~d}$ have AABR included as part of their screening so that neural hearing loss will not be missed (14).

Typically, the UNHS programs are a two-stage approach. First stage of screening is performed in the first days of life, before discharge from the hospital after birth and usually involves one or two-step OAE testing or OAE and AABR in high risk infants. Children who fail in-hospital screening test are referred for a repeat testing between 2 and $8 \mathrm{wk}$ after discharge (second stage) and are examined by means of OAE followed by AABR. Positive second stage results should be validated by otolaryngological and audiological consultation, diagnostic ABR testing and other electrophysiological testing performed by the third month of age. Finally, all infants identified with a hearing loss should receive appropriate early intervention very soon after the final diagnosis, and definitely before the 6 mo of age (9).

There is not one common UNHS protocol. The protocols vary with respect to the age of the newborn at first screening and the use of multiple tests either with repeat OAE tests or with the AABR test performed on those who fail the initial OAE test. The JCIH recommends that each country should adopt the most important rules to create the own protocol according to regional administrative possibilities, governmental decisions and budget (Table 3) (14).

Table 2. Otoacoustic emissions (OAEs) and automated auditory brain stem responses (AABR)—comparison of the techniques used in the universal hearing screening programs

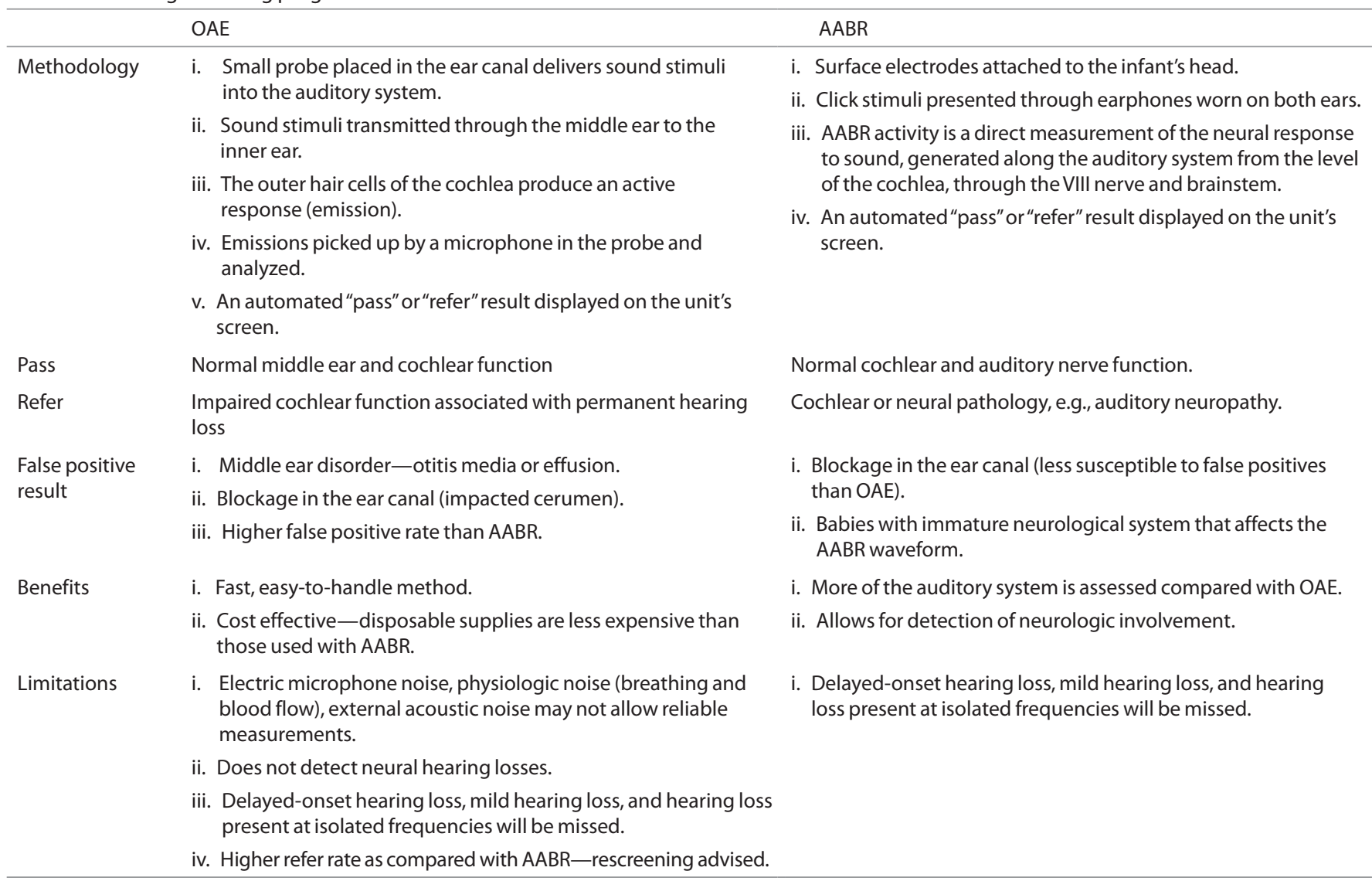


Table 3. Screening protocols at first level of universal newborn hearing screening programs in different countries all over the world

\begin{tabular}{|c|c|c|c|}
\hline Country & Since when & Time of screening & Screening method \\
\hline Austria (61) & 1995; 2003-data collection & Day 2-3 & $O A E+A A B R$ \\
\hline Spain (62) & 1998 & Day 2-3 & $\begin{array}{l}\text { two-step OAE/OAE + AABR } \\
\text { if known risk factor }\end{array}$ \\
\hline Finland (63) & 2000 & Before discharge from the hospital & OAE or $A A B R$ \\
\hline Oman (64) & 2001 & Day 2-3 & OAE \\
\hline Poland $(47,48)$ & 2002 & Day 2-3 & OAE \\
\hline The Netherlands (66) & 2003 & $<2 w k$ & $2 O A E+A A B R$ \\
\hline Nigeria (59) & 2005 & Day 2-3 & $\mathrm{OAE}+\mathrm{AABR}$ \\
\hline Denmark (67) & 2005 & Day 4-10 & OAE \\
\hline United Kingdom (13) & 2006 & Day $>2$ & $O A E+A A B R$ \\
\hline Norway (68) & 2006 & Day 2-3 & OAE \\
\hline Brazil $(64,70)$ & 2007 & Before discharge from the hospital & OAE \\
\hline Russia (64) & 2008 & & OAE \\
\hline Germany (64) & 2009 & Day 2-3 & $\begin{array}{l}O A E+A A B R \text { or } A A B R \\
\text { if known risk factors }\end{array}$ \\
\hline Korea (64) & 2009 & Within $30 \mathrm{~d}$ & Various protocols \\
\hline Philippines (64) & 2009 & $\begin{array}{l}\text { Before discharge from the hospital or } \\
\text { within } 3 \text { mo of life }\end{array}$ & OAE or AABR \\
\hline Italy (Umbria) (71) & 2010 & Before discharge & $O A E+A A B R$ \\
\hline USA (72) & & Day $>2$ & $\mathrm{OAE}+\mathrm{AABR}$ or $\mathrm{AABR}$ \\
\hline China (64) & & Various protocols & $\begin{array}{c}\text { OAE in well-baby nursery, } \\
\text { AABR in NICU }\end{array}$ \\
\hline
\end{tabular}

AABR, automated auditory brain stem responses; OAE OAEs, otoacoustic emissions.

OAE — only OAE test at first level of screening.

$\mathrm{OAE}+\mathrm{AABR}$ - all children are tested with OAE and with AABR

Screening protocols at the first stage of the UNHS programs can be classified into four categories:

1. AABR only-both neural and cochlear hearing losses are detected using one type of technology; can be used in NICU and in well-infant nursery

2. OAEs only-recommended for use in well-infant nursery; do not detect neural hearing losses; high refer rates

3. OAE followed by AABR when the OAE is not passedOAE screening is completed on both ears first, AABR is only done for those newborns that do not pass the OAE screen. If one or both ears do not pass the AABR, the infant is referred for outpatient diagnostic testing

4. Both AABR and OAE-newborns must pass both an $\mathrm{OAE}$ and an AABR screening. The newborn who fails one or both screenings in one or both ears, is referred for outpatient diagnostic testing. The most precise but also the most expensive protocol; high fail rate.

\section{BENEFITS}

It is commonly accepted that early identification of hearing loss gives children the opportunity to develop significantly improved language skills compared with those children who are diagnosed later $(4,13,15,16,51)$. Children identified with hearing deficit before 6 mo of age may not have any speech and language delays and develop equally to their hearing peers in terms of speech and language (7).

Prior to newborn hearing screening, the average age of diagnosis of hearing impairment that compromised speech and language development was $26 \mathrm{mo}$, with hearing aid fitting at 32.2 mo (15). Many of these children failed to acquire good speech and oral language as the late start meant that the critical period for speech and language acquisition had passed.

The primary aim of early intervention in children with hearing impairment is to restore or promote the child's communication skills and do optimize the level of language development, which impacts the cognitive and socioemotional behavior $(10,15,51)$. Two main types of assistive devices are currently used to improve auditory perception: amplification devices and cochlear implants.

Amplification devices or hearing aids are used to intensify incoming sounds so that their loudness falls within the useful dynamic range of the patient. Cochlear implants on the contrary are used to restore useful hearing in bilateral severe to profound hearing loss for which amplification does not allow 


\section{Review | Wroblewska-Seniuk et al.}

satisfactory progress in auditory skills and spoken language. The best results of cochlear implants in congenital cases of hearing loss are obtained in early implantation, before the age of 6 mo $(10,15,51)$, hence the worldwide efforts in favor of UNHS programs. The longer the diagnosis and intervention delay, the poorer the results in terms of speech, language, social-emotional and cognitive development levels, regardless of comorbidity and additional disabilities $(15,51)$.

The benefit-cost analysis of UNHS shows that it is a worthwhile investment for society as benefits considerably outweigh the drawbacks and costs that are associated with the screening program. Early diagnosis saves the costs of intensive speech and language intervention and special education services $(39,49,51)$. Keren et al. in their study concluded that since early identification and intervention of a hearing disorder leads to better language skills, lower educational costs and increased lifetime productivity, the cost-effectiveness of universal neonatal hearing screening is much better than no screening or selective screening (52). Schroeder et al. found that mean yearly societal cost in children with hearing impairment was three times higher than the cost of normally hearing children at 7-9 $y$ of age. The severity of the hearing loss was an important predictor of the societal costs, such as special education services, adaptations required by children with medical disabilities and lost productivity by parents. They estimated that UNHS could reduce these costs considerably and showed that the best estimate of the annual cost saving in middle childhood is $21 \%$ of the neonatal cost of UNHS per one child identified with hearing impairment (53).

It must be underlined that screening for hearing loss fulfills all the criteria for universal screening. First of all, its prevalence is very high. Secondly, if it is not diagnosed early and managed appropriately, it has severe consequences for the affected child and its family. And finally, the techniques used to diagnose this problem are relatively inexpensive, easily available, accurate, objective, and reliable (7). It should be pointed out that prior to the universal hearing screening, the severe hearing loss was diagnosed on average at the age of 2-3y and mild-to-moderate hearing loss was often not identified until $4 \mathrm{y}$ of age (7). Over the years since UNHS programs have been implemented, the mean time of hearing loss detection became shorter and for most children it is now the age of several months. According to the $\mathrm{JCIH}$, the infant hearing screening should be performed within the first month of age, comprehensive audiological assessment should be accomplished before 3 mo of age and appropriate intervention should begin before 6 mo of age (14).

\section{PROBLEMS}

Newborn hearing screening does not always identify hearing deficits in infants, which means that the children pass screening although in fact suffer from hearing deficit. In such situation the diagnosis is often delayed due to false reassurance by the negative testing in the first days of life. The main causes of late-onset deafness are cytomegalovirus infection, genetic syndromes associated with progressive hearing loss, neurodegenerative disorders, trauma and bacterial meningitis in neonatal period. All these pathologies however are known to be risk factors for hearing loss and therefore, children in whom they are recognized should be referred to the second stage of screening at the age of $4-8 \mathrm{wk}$, regardless of the results of first-stage testing $(3,23,32,38,47,48)$.

Similarly, not recognized in the first stage of screening might be children with auditory neuropathy. In this disease the child passes OAE tests but has abnormal AABR. In countries where universal screening is based on OAE tests only and if there are not any risk factors, children are not referred for AABR exam and are not diagnosed in a timely manner. However, most cases of auditory neuropathy are high risk neonates, treated in the neonatal intensive care units and therefore it is now recommended that children admitted to NICU for more than $5 \mathrm{~d}$ should always be examined with combination of OAE and AABR either at first or second stage of screening $(14,54)$. Infants who do not pass AABR testing in the NICU should be referred directly to an audiologist for rescreening and comprehensive evaluation (14).

It is also now recommended that in all infants readmitted to the hospital in the first month of life, when there are conditions associated with potential hearing loss (e.g., hyperbilirubinemia, culture-positive sepsis, bacterial meningitis or treatment with ototoxic drugs), a repeat hearing screening is performed before discharge (14). The universal hearing screening, particularly if performed by means of OAE only, often leads to a large number of false positive tests $(9,47,48)$, meaning that all these children should be retested. This significantly increases the cost of the program and causes that children with false positive tests go through a prolonged unnecessary diagnostic procedure $(48,55,56)$.

Another significant weakness of UNHS programs is the percentage of newborns that did not pass the initial testing but did not have any further testing and were lost to follow-up. In some populations even $50 \%$ of children who failed hearing screening may be missed to follow-up (9,55-58). It seems that the most important factor determining this situation is lack of parental involvement, such as delayed and missed appointments and reluctance for evaluations and interventions $(7,48)$. Sometimes parents' attitudes are due to their concern about stigma associated with hearing aids that results in delayed and less frequent use of the devices which compromises their effectiveness $(14,59)$. Socioeconomic factors also have significant influence on the effectiveness of hearing screening programs, especially in the developing countries (59).

The last but not least to be mentioned is, that mild hearing deficit between 20 and $35 \mathrm{~dB}$ is usually missed in hearing screening regardless of the protocol involved. However, such a hearing impairment has probably no serious impact on the child's well-being (9).

\section{THE FUTURE}

The main problems concerning universal hearing screening to be resolved are the following:

1. The initial referral rate should be significantly decreased and should not exceed $4 \%$, as set by the American 
Academy of Pediatrics and suggested by other guidelines $(1,14,60)$

2. The unacceptable high rate of lost-to-follow-up infants should be substantially reduced. A dedicated administrative system is of crucial importance in order to follow up each failed newborn and remind parents of their future appointments $(9,60)$

3. The gold standard of UNHS should be nationwide screening, prearranged according to the guidelines of the JCIH. Programs should guarantee close cooperation between maternity units and audiological centers in order to decrease time between exams, eliminate unnecessary tests, and assure proper interventions

4. The combination of OAE and AABR testing, which provides the most accurate diagnosis of hearing loss and significantly reduces the referral rate, should be considered to be in the future a standard protocol in newborn hearing screening.

\section{STATEMENT OF FINANCIAL SUPPORT}

No financial assistance was received to support this study.

Disclosure: The authors of this paper have no conflicts of interest relevant to this article to disclose.

\section{REFERENCES}

1. Erenberg A, Lemons J, Sia C, Trunkel D, Ziring P. Newborn and infant hearing loss: detection and intervention. American Academy of Pediatrics. Task Force on Newborn and Infant Hearing, 1998-1999. Pediatrics 1999;103:527-30.

2. Mehl AL, Thomson V. Newborn hearing screening: the great omission. Pediatrics 1998;101:E4.

3. Yoon PJ, Price M, Gallagher K, Fleisher BE, Messner AH. The need for long-term audiologic follow-up of neonatal intensive care unit (NICU) graduates. Int J Pediatr Otorhinolaryngol 2003;67:353-7.

4. Yoshinaga-Itano C. Benefits of early intervention for children with hearing loss. Otolaryngol Clin North Am 1999;32:1089-102.

5. Hess M, Finckh-Krämer U, Bartsch M, Kewitz G, Versmold H, Gross M. Hearing screening in at-risk neonate cohort. Int J Pediatr Otorhinolaryngol 1998;46:81-9.

6. Hille ET, van Straaten HI, Verkerk PH; Dutch NICU Neonatal Hearing Screening Working Group. Prevalence and independent risk factors for hearing loss in NICU infants. Acta Paediatr 2007;96:1155-8.

7. Nikolopoulos TP. Neonatal hearing screening: what we have achieved and what needs to be improved. Int J Pediatr Otorhinolaryngol 2015;79:635-7.

8. Biernath KR, Montero DP, Mehl A, Toomey KE. Universal newborn hearing screening and beyond. Am Fam Physician 2010;81:124.

9. Papacharalampous GX, Nikolopoulos TP, Davilis DI, Xenellis IE, Korres SG. Universal newborn hearing screening, a revolutionary diagnosis of deafness: real benefits and limitations. Eur Arch Otorhinolaryngol 2011;268:1399-406.

10. Deltenre P, Van Maldergem L. Hearing loss and deafness in the pediatric population: causes, diagnosis, and rehabilitation. Handb Clin Neurol 2013;113:1527-38.

11. Lieu JE. Speech-language and educational consequences of unilateral hearing loss in children. Arch Otolaryngol Head Neck Surg 2004;130:524-30.

12. Joint Committee on Infant Hearing, American Academy of Audiology, American Academy of Pediatrics, et al. Year 2000 position statement: principles and guidelines for early hearing detection and intervention programs. Pediatrics 2000;106:798-817.

13. Pimperton $\mathrm{H}$, Kennedy CR. The impact of early identification of permanent childhood hearing impairment on speech and language outcomes. Arch Dis Child 2012;97:648-53.
14. Joint Committee on Infant Hearing. Year 2007 position statement: principles and guidelines for early hearing detection and intervention programs. Pediatrics 2007;120:898-921.

15. Yoshinaga-Itano C. Early intervention after universal neonatal hearing screening: impact on outcomes. Ment Retard Dev Disabil Res Rev 2003;9:252-66

16. Yoshinaga-Itano C, Coulter D, Thomson V. Developmental outcomes of children with hearing loss born in Colorado hospitals with and without universal newborn hearing screening programs. Semin Neonatol 2001;6:521-9.

17. Steel KP, Kros CJ. A genetic approach to understanding auditory function. Nat Genet 2001;27:143-9.

18. Rehm HL. A genetic approach to the child with sensorineural hearing loss. Semin Perinatol 2005;29:173-81.

19. De Leenheer EM, Janssens S, Padalko E, Loose D, Leroy BP, Dhooge IJ. Etiological diagnosis in the hearing impaired newborn: proposal of a flow chart. Int J Pediatr Otorhinolaryngol 2011;75:27-32.

20. Vos B, Senterre C, Lagasse R, Levêque A; SurdiScreen Group. Newborn hearing screening programme in Belgium: a consensus recommendation on risk factors. BMC Pediatr 2015;15:160.

21. Leal MC, Muniz LF, Ferreira TS, et al. Hearing loss in infants with microcephaly and evidence of congenital Zika virus infection-Brazil, November 2015-May 2016. MMWR Morb Mortal Wkly Rep 2016;65:917-9.

22. American Academy of Audiology. Zika virus disease outbreak and infant hearing loss. 2016. http://www.audiology.org/news/zika-virusdisease-outbreak-and-infant-hearing-loss.

23. Cristobal R, Oghalai JS. Hearing loss in children with very low birth weight: current review of epidemiology and pathophysiology. Arch Dis Child Fetal Neonatal Ed 2008;93:F462-8.

24. Fausti SA, Henry JA, Helt WJ, et al. An individualized, sensitive frequency range for early detection of ototoxicity. Ear Hear 1999;20:497-505.

25. Williams AL, van Drongelen W, Lasky RE. Noise in contemporary neonatal intensive care. J Acoust Soc Am 2007;121(5 Pt1):2681-90.

26. Ranganath R. Reducing noise on the neonatal unit. Infant 2011; 7: $25-28$.

27. American Academy of Pediatrics. Committee on Environmental Health Noise: a hazard for the fetus and newborn. Pediatrics 1997;100:724-27.

28. Shapiro SM, Popelka GR. Auditory impairment in infants at risk for bilirubin-induced neurologic dysfunction. Semin Perinatol 2011;35:162-70.

29. Shapiro SM, Nakamura H. Bilirubin and the auditory system. J Perinatol 2001;21 Suppl 1:S52-5; discussion S9-62.

30. Haupt H, Scheibe F, Ludwig C. Changes in cochlear oxygenation, microcirculation and auditory function during prolonged general hypoxia. Eur Arch Otorhinolaryngol 1993;250:396-400

31. Robertson CM, Howarth TM, Bork DL, Dinu IA. Permanent bilateral sensory and neural hearing loss of children after neonatal intensive care because of extreme prematurity: a thirty-year study. Pediatrics 2009;123:e797-807.

32. D'Agostino JA, Austin L. Auditory neuropathy: a potentially under-recognized neonatal intensive care unit sequela. Adv Neonatal Care 2004;4: 344-53.

33. Downs MP, Sterritt GM. Identification audiometry for neonates: a preliminary report. J Aud Res 1964;4:69-80.

34. Downs MP, Yoshinaga-Itano C. The efficacy of early identification and intervention for children with hearing impairment. Pediatr Clin North Am 1999;46:79-87.

35. Northern JL. Marion downs: her life and legacy (1914-2014). Int J Audiol 2015;54:289-90.

36. Joint Committee on Infant Hearing. 1994 position statement. ASHA 1994;36:38-41.

37. Bolisetty S, Dhawan A, Abdel-Latif M, Bajuk B, Stack J, Lui K; New South Wales and Australian Capital Territory Neonatal Intensive Care Units' Data Collection. Intraventricular hemorrhage and neurodevelopmental outcomes in extreme preterm infants. Pediatrics 2014;133:55-62.

38. Coenraad S, Goedegebure A, van Goudoever JB, Hoeve LJ. Risk factors for sensorineural hearing loss in NICU infants compared to normal hearing NICU controls. Int J Pediatr Otorhinolaryngol 2010;74:999-1002. 
39. Thompson DC, McPhillips H, Davis RL, Lieu TL, Homer CJ, Helfand M. Universal newborn hearing screening: summary of evidence. JAMA 2001;286:2000-10.

40. Sokol J, Hyde M. Hearing screening. Pediatr Rev 2002;23:155-62.

41. Norton SJ, Gorga MP, Widen JE, et al. Identification of neonatal hearing impairment: evaluation of transient evoked otoacoustic emission, distortion product otoacoustic emission, and auditory brain stem response test performance. Ear Hear 2000;21:508-28.

42. Foust T, Eiserman W, Shisler L, Geroso A. Using otoacoustic emissions to screen young children for hearing loss in primary care settings. Pediatrics 2013;132:118-23.

43. Janssen T. A review of the effectiveness of otoacoustic emissions for evaluating hearing status after newborn screening. Otol Neurotol 2013;34:105863.

44. Richardson MP, Williamson TJ, Reid A, Tarlow MJ, Rudd PT. Otoacoustic emissions as a screening test for hearing impairment in children recovering from acute bacterial meningitis. Pediatrics 1998;102:1364-8.

45. White KR, Vohr BR, Maxon AB, Behrens TR, McPherson MG, Mauk GW. Screening all newborns for hearing loss using transient evoked otoacoustic emissions. Int J Pediatr Otorhinolaryngol 1994;29:203-17.

46. Eiserman WD, Hartel DM, Shisler L, Buhrmann J, White KR, Foust T. Using otoacoustic emissions to screen for hearing loss in early childhood care settings. Int J Pediatr Otorhinolaryngol 2008;72:475-82.

47. Wroblewska-Seniuk K, Chojnacka K, Pucher B, Szczapa J, Gadzinowski J, Grzegorowski M. The results of newborn hearing screening by means of transient evoked otoacoustic emissions. Int J Pediatr Otorhinolaryngol 2005;69:1351-7.

48. Szyfter W, Wróbel M, Radziszewska-Konopka M, Szyfter-Harris J, Karlik M. Polish universal neonatal hearing screening program-4-year experience (2003-2006). Int J Pediatr Otorhinolaryngol 2008;72:1783-7.

49. Akinpelu OV, Peleva E, Funnell WR, Daniel SJ. Otoacoustic emissions in newborn hearing screening: a systematic review of the effects of different protocols on test outcomes. Int J Pediatr Otorhinolaryngol 2014;78:711-7.

50. Avan P, Büki B, Maat B, Dordain M, Wit HP. Middle ear influence on otoacoustic emissions. I: noninvasive investigation of the human transmission apparatus and comparison with model results. Hear Res 2000;140: 189-201.

51. Yoshinaga-Itano C. Levels of evidence: universal newborn hearing screening (UNHS) and early hearing detection and intervention systems (EHDI). J Commun Disord 2004;37:451-65.

52. Keren R, Helfand M, Homer C, McPhillips H, Lieu TA. Projected costeffectiveness of statewide universal newborn hearing screening. Pediatrics 2002;110:855-64.

53. Schroeder L, Petrou S, Kennedy C, et al. The economic costs of congenital bilateral permanent childhood hearing impairment. Pediatrics 2006;117:1101-12.

54. Berg AL, Spitzer JB, Towers HM, Bartosiewicz C, Diamond BE. Newborn hearing screening in the NICU: profile of failed auditory brainstem response/passed otoacoustic emission. Pediatrics 2005;116:933-8.

55. Clemens CJ, Davis SA, Bailey AR. The false-positive in universal newborn hearing screening. Pediatrics 2000;106:E7.

56. Lin HC, Shu MT, Lee KS, Lin HY, Lin G. reducing false positives in newborn hearing screening program: how and why. Otol Neurotol 2007;28:788-92.
57. Vos B, Lagasse R, Levêque A. Main outcomes of a newborn hearing screening program in Belgium over six years. Int J Pediatr Otorhinolaryngol 2014;78:1496-502.

58. Rohlfs AK, Wiesner $\mathrm{T}$, Drews $\mathrm{H}$, et al. Interdisciplinary approach to design, performance, and quality management in a multicenter newborn hearing screening project: introduction, methods, and results of the newborn hearing screening in Hamburg (Part I). Eur J Pediatr 2010;169: 1353-60.

59. Olusanya BO. Neonatal hearing screening and intervention in resourcelimited settings: an overview. Arch Dis Child 2012;97:654-9.

60. Rohlfs AK, Wiesner T, Drews $\mathrm{H}$, et al. Interdisciplinary approach to design, performance, and quality management in a multicenter newborn hearing screening project. Discussion of the results of newborn hearing screening in Hamburg (part II). Eur J Pediatr 2010;169:1453-63.

61. Weichbold V, Nekahm-Heis D, Welzl-Mueller K. Ten-year outcome of newborn hearing screening in Austria. Int J Pediatr Otorhinolaryngol 2006;70:235-40.

62. Granell J, Gavilanes J, Herrero J, Sánchez-Jara JL, Velasco MJ, Martín G. [Is universal newborn hearing screening more efficient with auditory evoked potentials compared to otoacoustic emissions?]. Acta Otorrinolaringol Esp 2008;59:170-5.

63. Ministry of Social Affairs and Health. Screenings in Finland 2014. The present state of health care screenings and future prospects. 2014. https:// www.julkari.fi/bitstream/handle/10024/126998/STM_Screenings_i_finland_2014_Enkku_B5_nettiin.pdf?sequence $=1$.

64. Newborn and infant hearing screening. Current issues and guiding principles for action. Outcome of a WHO informal consultation held at who headquarters, Geneva, Switzerland, 09-10 November 2009. http://www. who.int/blindness/publications/Newborn_and_Infant_Hearing_Screening_Report.pdf.

65. Prpić I, Mahulja-Stamenković V, Bilić I, Haller H. Hearing loss assessed by universal newborn hearing screening-the new approach. Int J Pediatr Otorhinolaryngol 2007;71:1757-61.

66. Korver AM, Konings S, Dekker FW, et al.; DECIBEL Collaborative Study Group. Newborn hearing screening vs. later hearing screening and developmental outcomes in children with permanent childhood hearing impairment. JAMA 2010;304:1701-8.

67. Konradsson KS, Kjaerboel E, Boerch K. Introducing universal newborn hearing screening in Denmark: preliminary results from the city of Copenhagen. Audiological Medicine. 2007;5:176-81.

68. Elverland HH. Infants who cannot hear. Tidsskr Nor Laegeforen 2015;135:103.

69. Hergils L. Analysis of measurements from the first Swedish universal neonatal hearing screening program. Int J Audiol 2007;46:680-5.

70. Bevilacqua MC, Alvarenga Kde F, Costa OA, Moret AL. The universal newborn hearing screening in Brazil: from identification to intervention. Int J Pediatr Otorhinolaryngol 2010;74:510-5.

71. Molini E, Calzolaro L, Lapenna R, Ricci G. Universal newborn hearing screening in Umbria region, Italy. Int J Pediatr Otorhinolaryngol 2016;82:92-7.

72. White KR, Forsman I, Eichwald J, Munoz K. The evolution of early hearing detection and intervention programs in the United States. Semin Perinatol 2010;34:170-9. 